\title{
DICOM IMAGE COMPRESSION BASED ON SPATIAL FUZZY CLUSTERING USING WAVELET BASED CONTOURLET TRANSFORM
}

\author{
Rupa Sajin ${ }^{1}$ \\ ${ }^{1}$ Assistant Professor, Electronics and Communication, T. John Institute of Technology, Karnataka, India
}

\begin{abstract}
Medical imaging has a great impact on diagnosis, so high resolution is mandatory for the representation of such nontrivial images. On the other hand coding and transmission of medical images by preserving the clinically nontrivial information with reduction in storage space differ from standard image coding as it incorporates the integrity of CROI and bandwidth. The main concern in medical image compression lies in compressing a huge amount of visual data into a low bit rate stream. The proposed strategy intends to safeguard the clinically basic data with change in pressure proportion $(C R)$ and crest sign to commotion proportion (PSNR) In the proposed work altered spatial introduction tree (MSPIHT) is utilized to encode the wavelet based contourlet coefficients of the clinical area of interest (CROI) and SPIHT to encode the wavelet coefficients for encoding the foundation, portioned utilizing spatial fluffy C implies bunching (SFCM).The proposed calculation indicates change in PSNR and give effective representation of edges in DICOM (Digital Imaging and Communications in Medicine) pictures.
\end{abstract}

Key Words: DICOM Images, SFCM, Clinical Region of Interest, WBCT and Modified SPIHT

\section{INTRODUCTION}

PACS (Picture Archiving and Communication Systems) depends on medical image compression which plays a critical and vital role in the field of Telemedicine. The transfer of imaging reports of patients supported by telemedicine technology taken from different advanced imaging modalities such as Magnetic Resonance Imaging (MRI), Positron Emission Tomography (PET), Computed Tomography (CT), Ultrasounds (US), X-rays. Medical imaging is a technique which creates images of the human body for clinical diagnostic purpose. Advanced imaging modalities such as Sky Scan $2011 \mathrm{x}$-ray nano tomography has an image resolution of $200 \mathrm{~nm}$ per pixel, micro computed tomography (CT) reconstructs images with 8000 x 8000 pixels per slice which occupies 64MB storage space per slice and Polarized light imaging (PLI) reconstructs images with 3569 × 2700 pixels which needs 47.4GB amount of memory for storage of all scanned images.

To preserve the clinically important characteristics in medical imaging the input image is segmented into meaningful regions. Numerical algorithms have been proposed for DICOM image segmentation which includes local edge detection, morphological region based approach, threshold method, histogram methods and clustering approaches. In order to predict the critically clinical region of interest clustering techniques are most widely used in biomedical applications. Unsupervised method such as fuzzy based clustering approaches are widely used for the segmentation of medical images. Clustering of medical data poses difficulties mainly in terms of spatial and temporal components of the data [1]. Recently DICOM images are compressed using wavelet based methods. In order to overcome the missing directionality of conventional transforms, Multiresolution Geometric Analysis (MRA) [2] such as Bandlet, Brushlet, Contourlet, Curvelet, Ridgelet, Pearlet and so on for high dimensional signals have been developed.

In this algorithm, WBCT a new family of geometrical image transform that decomposes images both radially and angularly is used. WBCT comprises of two stages of filter banks, non redundant and perfect reconstruction. WBCT is visually superior than wavelet coder and produces good PSNR rate curves than wavelet coders.

\subsection{Literature Survey}

E. A. Zanaty and Ashraf Afifi have proposed [3] an automatic fuzzy clustering for magnetic resonance image clustering. Their proposed work produces six clusters with an clustering accuracy of 0.823 and seven clusters with an clustering accuracy of 0.743 . S. Patel and K.S. Patnaik have proposed [4] a clustering algorithm for MR Image segmentation using IQI. They have worked on RFCM (Rough Fuzzy C Means) clustering and their algorithm is analysed on T1 and T2-weighted MRI image for the same patient. The images are divided into six clusters their results were proved to have less noise in segmentation compared to conventional FCM clustering.

Abdul Khader Jilani Saudagar and Abdul Sattar Syed have proposed [5] compression of biomedical images using ridgelet transform and modified neuro modelling. Their results are found to be better compared to JPEG2000. WenJyi Hwang, Ching-Fung Chine and Kup-Jung Li have proposed [6] a scalable medical data compression using wavelet transform for telemedicine applications. They have analysed their proposed method on MR images and have 
compared their result with JPEG2000 and SPIHT algorithm and SNR of the proposed LSPIHT algorithm found to be better than those two conventional methods for MRI 01 LSPIHT has SNR of 35.81 JPEG2000 has 35.37 and SPIHT has $25.51 \mathrm{~dB}$ in terms of compression ratio. V.K. Sudha and R. Sudhakar have proposed [7] a work on multiwavelet transform for compressing medical images. They have proposed SSIM index as a quality measure for reconstructed image and the PSNR values of reconstructed images at various bitrates for different multiwavelet.

\section{REVIEW OF RELATED THEORY}

In order to achieve better segmentation pre-processing of the DICOM image is done for removing the noise. The critically clinical region of interest in the DICOM image is extracted using SFCM clustering technique in the proposed algorithm. The CROI is transformed using WBCT and MSPIHT is applied to encode the WBCT coefficients.

\subsection{Spatial Fcm}

The probability the pixels possessing similar feature values and the probability that they belong to the same cluster is an important characteristics of an image with its neighbor. This spatial relationship among pixels play a nontrivial role in clustering. SFCM proposed by Chuang et al [8] overcomes the inherent limitation of standard FCM as FCM algorithm does not utilize it. For clustering in SFCM the spatial information is incorporated in to the FCM membership function. In the neighborhood the membership weighting of each cluster is altered after the cluster. The clustering of SFCM at each iteration is a two-pass process.

\section{- $\quad$ First Pass:}

In the spectral domain calculation of membership function is done similar to that of conventional FCM.

FCM partitions collection of $\mathrm{m}$ vector into $\mathrm{C}$ fuzzy groups $x_{x}$; where $x=1,2, \ldots m$. The $(c \mathrm{x} n)$ binary partition matrix $U$ are typically defined; where the element $U_{i j}$ is 1 if the $j^{\text {th }}$ data point $x_{i}$ belongs to group $x$ or 0 otherwise. $U$ being a real matrix. $\mathrm{C}=c_{1}, c_{2}, \ldots c_{c} . \in=0.001$, stopping condition. $\mathrm{MI}=100$, maximum iteration

$$
\begin{aligned}
& U=\left[U_{x j}\right]_{x}\left(y_{k}\right)=U_{x j} \\
& \sum_{x=1}^{c} U_{x j}>0 \forall x \\
& \sum_{x=1}^{c} U_{x j}>0 \forall j=1,2, \ldots m
\end{aligned}
$$

The cost function which is to be minimized is defined by

$J\left(U, c_{1}, c_{2}, \ldots c_{c}\right)=\sum_{x=1}^{c} J_{x}$

$J\left(U, c_{1}, c_{2}, \ldots c_{c}\right)=\sum_{x=1}^{c} \sum_{j=1}^{n} U_{x j}^{n} d_{x j}^{2}$

$U_{x j}$ is between 0 and 1 .

$c_{x}$ is the cluster centre of fuzzy group $i$.

$d_{x j}=\left\|c_{x}-x_{j}\right\|$ is the Euclidean distance between $x^{t h}$ cluster centre and $j^{\text {th }}$ data point.

$$
\begin{aligned}
& \hat{J}\left(U, c_{1}, c_{2}, \ldots c_{c}, \lambda_{1}, \lambda_{2}, \ldots . \lambda_{n}\right)=J\left(U, c_{1}, c_{2}, \ldots c_{c}\right)+ \\
& \sum_{i=1}^{c} \lambda_{j}\left(\sum_{i=1}^{c} U_{i j}-1\right)
\end{aligned}
$$

where $\lambda_{j}$ is a Lagrange multipliers

Differentiating with respect to input arguments

$$
\begin{aligned}
& \hat{J}\left(U, c_{1}, c_{2}, \ldots c_{c}, \lambda_{1}, \lambda_{2}, \ldots . \lambda_{m}\right) \\
& c_{x}=\sum_{x=1}^{c} U_{x j}^{n} x_{x j} / \sum_{i=1}^{c} U_{x j}^{n} \\
& U_{x j}=\left[\sum_{k=1}^{c}\left(\frac{d_{x j}}{d_{k j}}\right)^{\frac{2}{m-1}}\right]^{-1}
\end{aligned}
$$

and cluster is given by

$$
V_{x}=\frac{\sum_{j=1}^{N} m X_{j}}{\sum_{j=1}^{N} U_{x j}^{n}}
$$

\section{- $\quad$ Second Pass:}

Membership information of each pixel is mapped on to the spatial domain for spatial function computation.

The spatial function is defined as

$h_{x j}=\sum_{k \in N B\left(x_{j}\right)} U_{x j}$

where $h_{x j}$ represents speaks to a square window fixated on pixel $x_{-} j$ in the spatial area. The spatial capacity is inbuilt into the participation capacity as take

$U_{x j}^{\prime}=\frac{U_{x j}^{p} h_{x j}^{q}}{\sum_{k=1}^{c} U_{x j}^{p} h_{x j}^{q}}$

where $\mathrm{p}$ and $\mathrm{q}$ controls the relative significance of both capacities.

\subsection{Wavelet Based Contourlet Transform}

WBCT proposed by Ramin Eslami and Hayder Radha is a new family of geometrical image transform that decompose images both radially and angularly [8]. WBCT comprises of two stages of filter banks which are non redundant and perfect reconstruction. WBCT is capable of approximating images texture and contours mostly [9]. At the first stage images are decomposed using wavelet transform using separable filter banks and in the second stage directional filter banks (DFB) (non separable filter banks) are applied on wavelet coefficients for angular decomposition so as to maintain the anisotropy scaling law.

\subsubsection{Multiresolution Analysis Of WBCT}

In an1-level DFB; $2^{\mathrm{s}}$ directional subbands are available with $\mathrm{G}_{\mathrm{i}}^{(\mathrm{s})}, 0 \leq \mathrm{i}<2^{\mathrm{s}}$ equivalent synthesis filters and the overall down sampling matrices of $\mathrm{S}_{\mathrm{i}}^{\mathrm{s})}, 0 \leq \mathrm{i}<2^{\mathrm{s}}$ is defined as

$\mathrm{S}_{\mathrm{i}}^{(\mathrm{s})}=\left[\begin{array}{cc}2(s-1) & 0 \\ 0 & 2\end{array}\right]$; if $0 \leq \mathrm{i}<2^{s-1}$

$\mathrm{Si}^{(\mathrm{s})}=\left[\begin{array}{cc}2 & 0 \\ 0 & 2(s-1)\end{array}\right] ;$ if $2^{s-1} \leq \mathrm{k}<2^{s}$

then $\left\{\mathrm{g}_{\mathrm{i}}^{\mathrm{s}}\left[\mathrm{n}-\mathrm{S}_{\mathrm{i}}^{(\mathrm{s})} \mathrm{m}\right]\right\}, 0 \leq \mathrm{i}<2^{s}, \mathrm{~m} \in \mathbb{Z}^{2}$ is a directional basis for $\ell^{2}\left(\mathbb{Z}^{2}\right) ; \mathrm{g}_{\mathrm{i}}^{\mathrm{s}}$ is the impulse response of the synthesis filter $\mathrm{G}_{\mathrm{i}}^{(\mathrm{s})}$. Asuming an orthonormal separable WT, separable @D multiresolution is given as [10].

$\mathrm{V}_{\mathrm{k}}^{2}=\mathrm{V}_{\mathrm{k}} \otimes \mathrm{V}_{\mathrm{k}}$ and $\mathrm{V}_{\mathrm{k}-1}^{2}=\mathrm{V}_{\mathrm{k}}^{2} \oplus \mathrm{W}_{\mathrm{k}}^{2}$ 
where $\mathrm{W}_{\mathrm{k}}^{2}$ is the detail space and orthogonal component of $\mathrm{V}_{\mathrm{k}}^{2}$ in $\mathrm{V}_{\mathrm{k}-1}{ }^{2}$. The family $\left\{\psi_{k, n}^{1}, \psi_{k, n}^{2}, \psi_{k, n}^{3}\right\}_{\mathrm{n}} \in \mathrm{z}^{2}$ is an orthonormal basis of $\mathrm{W}_{\mathrm{k}}^{2}$. On applying $\mathrm{s}_{\mathrm{k}}$ directional levels to the detail multiresolution space $\mathrm{W}_{\mathrm{k}}^{2}$.

$\mathrm{W}_{\mathrm{k}}^{2}=\bigoplus_{i=0}^{2^{s_{k-1}}} W_{k, i}^{2,\left(s_{k}\right)}$

Defining

$\eta_{i, k, n}^{i,\left(l_{k}\right)}=\sum_{m \in Z^{2}} g_{i}^{l_{k}}\left[\mathrm{~m}-S_{i}^{l_{k}} \mathrm{n}\right] \psi_{k, m}^{i} ; \mathrm{i}=1,2,3$.

The family $\left\{\eta_{i, k, n}^{1,\left(l_{k}\right)}, \eta_{i, k, n}^{2,\left(l_{k}\right)}, \eta_{i, k, n}^{3,\left(l_{k}\right)}\right\}_{\mathrm{n}} \in \mathrm{z}^{2}$ is a basis for the subspace $W_{k, i}^{2,\left(l_{k}\right)}$.

\subsection{Modified SPIHT}

DICOM images with straight singularities along with curve, contour and edges are encoded with an efficient adaptive subband coding algorithm in this proposed work. WBCT coefficients are scanned and encoded as much as possible to satisfy the demand of embedded coding algorithm. WBCT coefficients are effectively repositioned so that it could be encoded using SPIHT algorithm.

\section{PROPOSED COMPRESSION SCHEME}

The proposed compression scheme flow graph is illustrated in Fig 1. The various modules of the proposed scheme, using SFCM the input DICOM images are segmented [11] so that the critically clinical region of interest is extracted(CROI). In DICOM image segmentation the spatial relationship of neighboring pixel is an important characteristics [12]. WBCT is applied to the critically clinical region of interest segmented through SFCM. The WBCT decomposes the CROI using wavelet transform at the first stage and wavelet coefficients are filtered using directional filter banks.

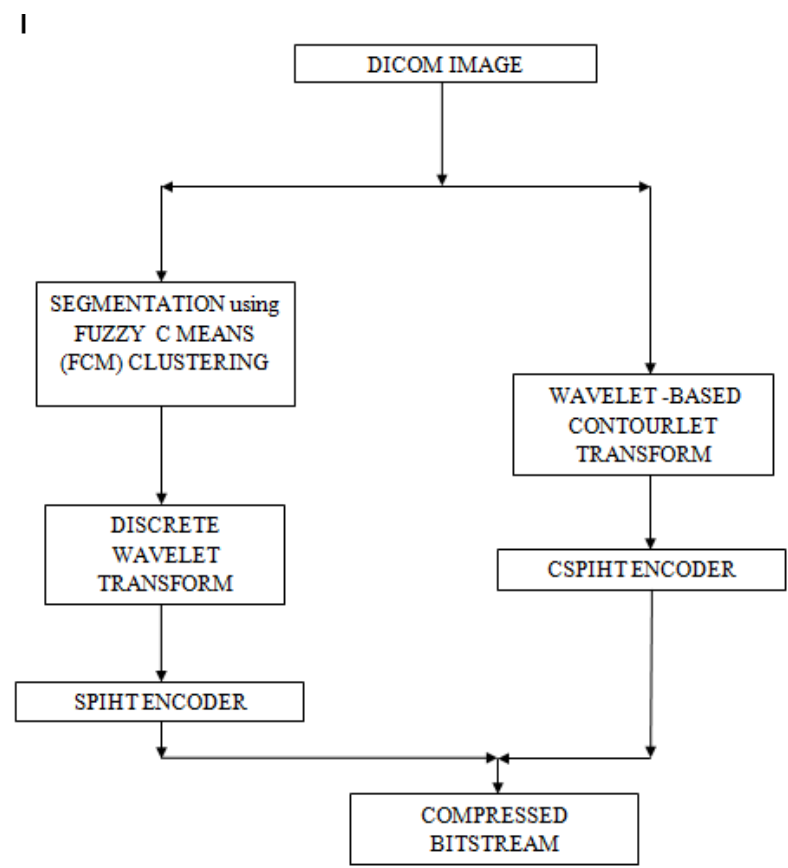

Fig -1: The proposed compression scheme flow diagram The WBCT transformed coefficients of critically clinical region of interest are repositioned so as to encode with spatial orientation trees as conventional wavelet coefficients. Wavelet coefficients of background are encoded with traditional SPIHT algorithm.

\section{PERFORMANCE EVALUATION}

The proposed algorithm is evaluated on set of six different DICOM images (MRI brain image - T2 flair axial of size, MRI brain image - T2W axial of size, MRI brain image T2_corflair axial of size, MRI thorax image of size, MRI brain tumor image of size, MRI brain lymphoma image of size) with 'dcm' (DICOM File Format) Cextension are taken as an input and is shown in Fig. 2. Quality assessment of the compressed images are done in terms of PSNR $(\mathrm{dB})$, Bitrate (bpp) and compression ratio fig 2 . The proposed algorithm efficiency is evaluated on comparison with JPEG, Wavelet and SFCM with curvelet based methods. 4 clusters outputs from SFCM are shown in following fig 3.
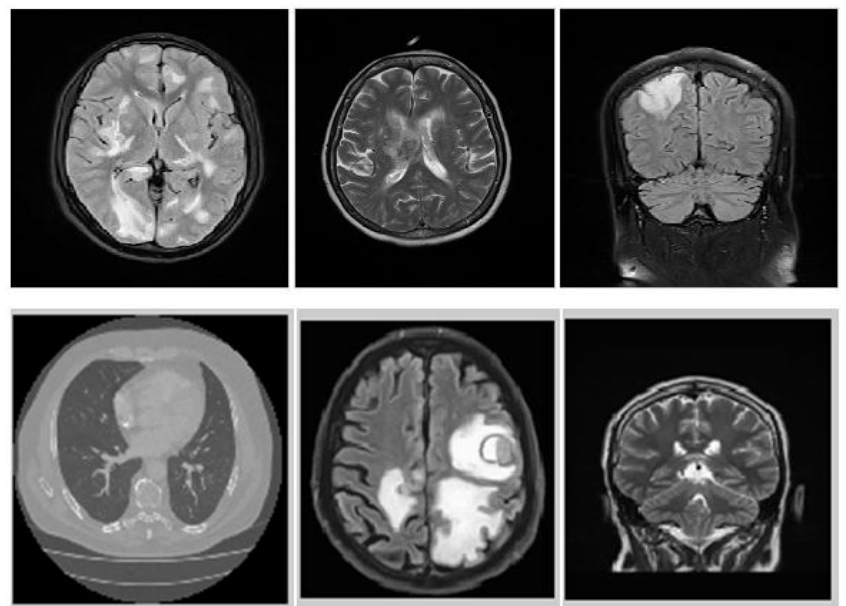

Fig. 2: Set of input images
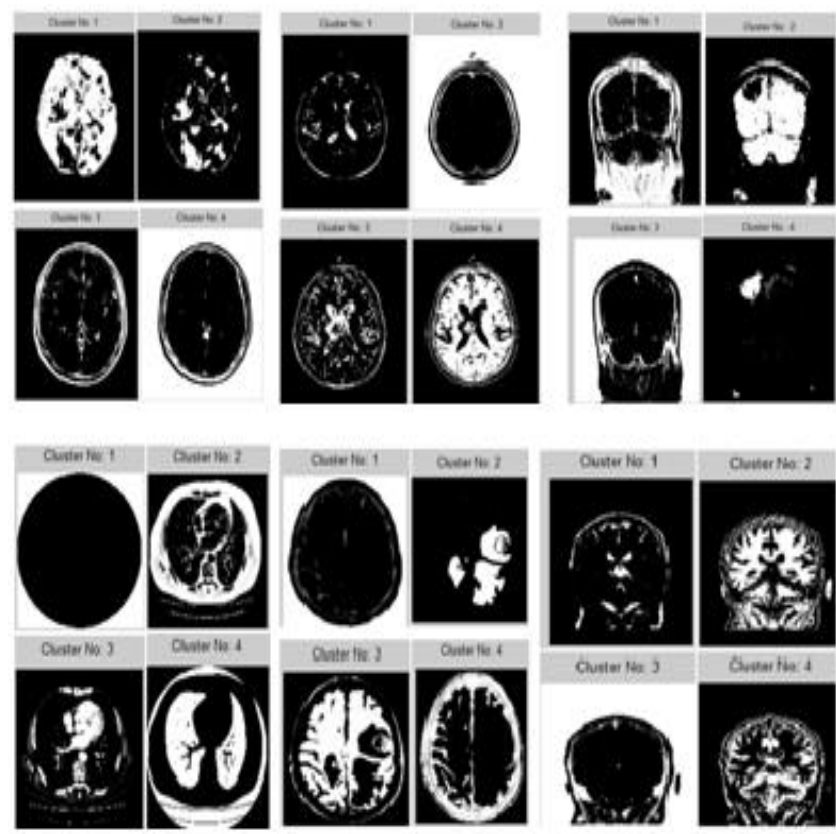

Fig. 3: Set of SFCM output images with 4 clusters 


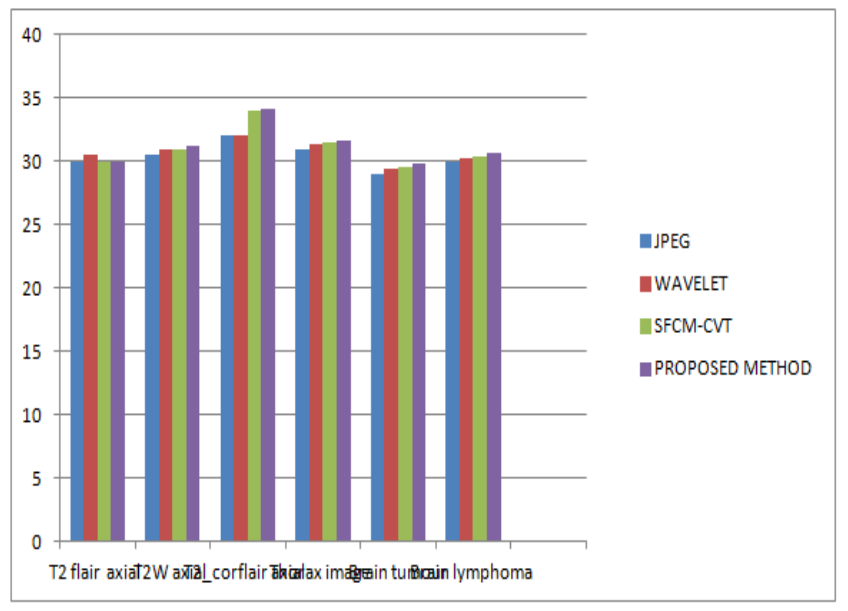

Chart -1: PSNR(dB) obtained for test images using various compression schemes

\section{CONCLUSIONS}

In the proposed paper WBCT based medical image compression for different DICOM images are implemented with modified SPIHT encoding algorithm. MSPIHT algorithm is based on traditional SPIHT algorithm but WBCT coefficients are repositioned before encoding with spatial orientation tree. As a future enhancement this work can be extended for video medical image processing. The simulation result of the proposed coder indicates that it is visually superior to other encoding methods in preserving detailed coefficients, contours and textures of medical images.

\section{REFERENCES}

[1]. Hesam Izakian, Witold Pedrycz and Iqbal Jamal: "Clustering Spatiotemporal Data: An Augmented Fuzzy C-Means"; IEEE Transactions On Fuzzy Systems, Vol. 21,No. 5, October 2013.

[2]. Peter Karasev, Ivan Kolesov, Karl Fritscher, Patricio Vela, Phillip and Allen Tannenbaum, "Interactive Medical Image Segmentation Using Control of Active Contours"; IEEE Transactions of Medical Imaging, Vol.32, No. 11, November 2013.

[3]. E. A. Zanaty and Ashraf Afifi: A New Approach For Automatic Fuzzy Clustering Applied To Magnetic Resonance Imaging Clustering; American Journal Of Remote Sensing, Vol. 1, No. 2, April 2013.Reference 3

[4]. S. Patel and K.S. Patnaik: "Analysis Of Clustering Algorithms For MR Image Segmentation Using IQI"; SciVerse Science Direct Procedia Technology 62012.

[5]. Abdul Khader Jilani Saudagar, Abdul Sattar Syed: "Image compression approach with ridgelet transformation using modified neuro modeling for biomedical images"; Springer-Verlag Neural Comput \& Applic DOI 10.1007/s00521-013-1414-y London April 2013.

[6]. Wen-jyi Hwang, Ching-fung Chine, And Kuo-jung Li: "Scalable Medical Data Compression And Transmission Using Wavelet Transform For Telemedicine Applications"; IEEE Transcations On
Infromation Technology In Biomedicine, Vol. 7, No. 1,march 2003.

[7]. V.K. Sudha and R. Sudhakar: "Multiwavelet Transform in Compression of Medical Images". ICTACT Journal on image and video processing, volume: 03, issue; 04, May 2013.

[8]. Ramin Eslami and Hayder Radha: " Wavelet-Based Contourlet Transform And Its Application To Image Coding"; IEEE International Conference on Image Processing, Singapore, Oct. 2004.

[9]. R. Eslami and H. Radha, "Wavelet-based contourlet coding using an SPIHT-like algorithm," in proceedings of Conference on Information Sciences and Systems, pp. 784-788, Princeton, March 2004.

[10].S. Mallat: " A Wavelet Tour Of Signal Processing, Academic Press, $2^{\text {nd }}$ Edition, 1998.

[11].Keh-Shih Chuang, Hong-Long Tzeng, Sharon Chen, Jay Wu, Tzong-Jer Chen: "Fuzzy C-Means Clustering With Spatial Information For Image Segmentation"; ELSEVIER Computerised Medical Imaging And Graphics 302003.

[12].S. Rupa, V. Mohan, Y. Venkataramani:"MRI Brain Image Compression using Spatial Fuzzy Clustering Technique"; IEEE International Conference on Communication and Signal Processing, April 3-5, 2014, India.

\section{BIOGRAPHIES}

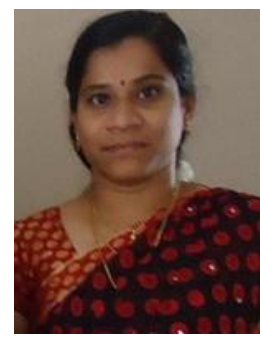

Rupa Sajin received her B.E and M.E degree from Saranathan College of Engineering, Tiruchirapalli, Tamil Nadu in 2004 and 2014. She is a university rank holder in her master degree. Her research interest includes image segmentation and compression. She has seven years of working experience. She is currently working as an assistant professor in department of ECE at T. John Institute of Technology, Bangalore, Karnataka. 